\title{
CERTAIN TWO-DIMENSIONAL MIXED BOUNDARY- VALUE PROBLEMS FOR WEDGE-SHAPED REGIONS AND DUAL INTEGRAL EQUATIONS
}

\author{
by R. P. SRIVASTAV
}

(Received 1st July 1964)

\section{Introduction}

Finding the distribution of stress in earth dams containing cracks is an outstanding problem of soil mechanics. Even the simplest mathematical model, viz., that of a wedge containing a plane crack which is symmetrically situated along the bisector plane of the angle of the wedge, with the plane strain assumption of the infinitesimal theory of elasticity, presents a difficult problem of solving the bi-harmonic equation subject to mixed boundary conditions. While elasticity problems related to wedge-shaped bodies have been investigated, it appears little attention has been paid to the mixed boundary-value problems. As a first step towards the solution of the mixed boundary value problem for the biharmonic equation, we discuss in this paper the solution of Laplace's equation

$$
\nabla^{2} \phi \equiv \frac{\partial^{2} \phi}{\partial \rho^{2}}+\frac{1}{\rho} \frac{\partial \phi}{\partial \rho}+\frac{1}{\rho^{2}} \frac{\partial^{2} \phi}{\partial \theta^{2}}+\frac{\partial^{2} \phi}{\partial z^{2}}=0
$$

for wedge-shaped regions subject to mixed type of conditions on the boundary. If we assume that $\phi$ does not depend on $z$, the equation (1.1) is reduced to the equation

$$
\frac{\partial^{2} \phi}{\partial \rho^{2}}+\frac{1}{\rho} \frac{\partial \phi}{\partial \rho}+\frac{1}{\rho^{2}} \frac{\partial^{2} \phi}{\partial \theta^{2}}=0 .
$$

We consider the solution of the equation (1.2) for the region $0<\rho<\infty$, $0<\theta<\alpha$, subject to two types of boundary conditions on the face $\theta=\alpha$. (a) In the first instance we suppose that for $\theta=\alpha, \phi(\rho, \theta)=\phi(\rho)$. (b) Secondly we assume that for $\theta=\alpha$, the normal derivative of $\phi(\rho, \theta)$ has the prescribed value $\phi_{1}(\rho)$. Corresponding to each of these two conditions, there can be two kinds of boundary conditions on the face $\theta=0$ :

I.

$$
\begin{aligned}
& \phi(\rho, 0)=f_{1}(\rho), \quad 0<\rho<1, \\
& \left.\frac{\partial \phi}{\partial \theta}\right|_{\theta=0}=f_{2}(\rho), \quad \rho>1 \text {. }
\end{aligned}
$$

E.M.S.-Y 
II.

$$
\begin{array}{cl}
\left.\frac{\partial \phi}{\partial \theta}\right|_{\theta=0} & =f_{1}(\rho), \quad 0<\rho<1, \\
\phi(\rho, 0) & =f_{2}(\rho), \quad \rho>1 .
\end{array}
$$

The functions $f_{1}(\rho)$ and $f_{2}(\rho)$ are known functions.

Using Mellin-transforms we easily find that a solution of (1.2) satisfying the condition (a) for an arbitrary choice of $\psi$ is

$$
\begin{aligned}
\phi(\rho, \theta) & =\frac{1}{2 \pi i} \int_{c-i \infty}^{c+i \infty} \psi(s) \sec \alpha s \sin (\alpha-\theta) s \rho^{-s} d s \\
& +\frac{1}{2 \pi i} \int_{c^{\prime}-i \infty}^{c^{\prime}+i \infty} \Phi(s) \sec \alpha s \cos (\alpha-\theta) s \rho^{-s} d s,
\end{aligned}
$$

where $\bar{\phi}(s)$ is the Mellin-transform of $\phi(\rho)$, and that a solution of (1.2) which satisfies the condition (b) is

$$
\begin{aligned}
\phi(\rho, \theta) & =\frac{1}{2 \pi i} \int_{c-i \infty}^{c+i \infty} \psi(s) \sec \alpha s \cos (\alpha-\theta) s \rho^{-s} d s \\
& -\frac{1}{2 \pi i} \int_{c^{\prime \prime}-i \infty}^{c+i \infty} s^{-1} \Phi_{1}(s) \sec \alpha s \sin (\alpha-\theta) s \rho^{-s} d s
\end{aligned}
$$

where $\overline{\phi_{1}}(s)$ is the Mellin-transform of $\phi_{1}(\rho)$.

The choice of $c, c^{\prime}$ and $c^{\prime \prime}$ is governed by the integrability of $\rho^{c-1} \phi(\rho, \theta)$, $\rho^{c^{\prime}-1} \phi(\rho), \rho^{c^{\prime \prime}-1} \phi_{1}(\rho)$ and $\psi(c+i t)$ and the property of their being of bounded variation but $\phi(\rho, \theta)$ and $\psi$ are unknown so that a sort of semi-inverse approach has to be made. We keep $c$ arbitrary and choose it to facilitate calculation. The boundary conditions on $\theta=0$ give us the following types of integral equations for determining the function $\psi(s)$ in each case:

$$
\begin{array}{lll}
\left(\mathrm{a}_{1}\right) & \frac{1}{2 \pi i} \int_{c-i \infty}^{c+i \infty} \psi(s) \tan \alpha s \rho^{-s} d s=f_{1}(\rho), & 0<\rho<1, \\
& \frac{1}{2 \pi i} \int_{c-i \infty}^{c+i \infty} s \psi(s) \rho^{-s} d s=-f_{2}(\rho), & \rho>1 . \\
\left(\mathrm{a}_{2}\right) & \frac{1}{2 \pi i} \int_{c-i \infty}^{c+i \infty} s \psi(s) \rho^{-s} d s=-f_{1}(\rho), & 0<\rho<1, \\
& \frac{1}{2 \pi i} \int_{c-i \infty}^{c+i \infty} \psi(s) \tan \alpha s \rho^{-s} d s=f_{2}(\rho), & \rho>1 . \\
\left(\mathrm{b}_{1}\right) & \frac{1}{2 \pi i} \int_{c-i \infty}^{c+i \infty} \psi(s) \rho^{-s} d s=f_{1}(\rho), & 0<\rho<1, \\
\frac{1}{2 \pi i} \int_{c-i \infty}^{c+i \infty} s \psi(s) \tan \alpha s \rho^{-s} d s=f_{2}(\rho), & \rho>1 .
\end{array}
$$


$\left(b_{2}\right)$

$$
\begin{array}{ll}
\frac{1}{2 \pi i} \int_{c-i \infty}^{c+i \infty} s \psi(s) \tan \alpha s \rho^{-s} d s=f_{1}(\rho), & 0<\rho<1, \\
\frac{1}{2 \pi i} \int_{c-i \infty}^{c+i \infty} \dot{\psi}(s) \rho^{-s} d s=f_{2}(\rho), & \rho>1 .
\end{array}
$$

For the sake of convenience in presentation, we assume in each of the above problems in the first instance that $f_{2}(\rho) \equiv 0$ and secondly that $f_{1}(\rho) \equiv 0$. Obviously the solution to the general problem can be constructed by adding the two solutions.

We reduce in each case the dual integral equations to a single equation of the second kind of Fredholm type. The technique is essentially the same as employed by Sneddon and Srivastav (1) for dual series equations. The analysis is formal throughout this paper and no attempt is made to justify the change of order of integration or differentiation within the integral sign.

The following elementary integrals occur frequently in our work:

$$
\begin{array}{ll}
\int_{0}^{t} \rho^{-s}\left(t^{2}-\rho^{2}\right)^{-\frac{1}{2}} d \rho=\frac{\Gamma\left(\frac{1}{2}-\frac{1}{2} s\right) \Gamma\left(\frac{1}{2}\right)}{2 \Gamma\left(1-\frac{1}{2} s\right)} t^{-s}, & R(s)<1 . \\
\int_{t}^{\infty} \rho^{-s}\left(\rho^{2}-t^{2}\right)^{-\frac{1}{2}} d \rho=\frac{\Gamma\left(\frac{1}{2} s\right) \Gamma\left(\frac{1}{2}\right)}{2 \Gamma\left(\frac{1}{2}+\frac{1}{2} s\right)} t^{-s}, & R(s)>0 .
\end{array}
$$

We have also used the inversion theorem for Mellin-transform for which reference may be made to (2).

2. We begin our discussion of the dual integral equations given in $\S 1$, by considering the pair of equations

$$
\begin{aligned}
& \frac{1}{2 \pi i} \int_{c-i \infty}^{c+i \infty} \psi(s) \tan \alpha s \rho^{-s} d s=f_{1}(\rho), \quad 0<\rho<1, \\
& \frac{1}{2 \pi i} \int_{c-i \infty}^{c+i \infty} s \psi(s) \rho^{-s} d s=0, \quad \rho>1 ;-1<c<1 .
\end{aligned}
$$

If we assume that for $0<\rho<1$,

$$
\frac{1}{2 \pi i} \int_{c-i \infty}^{c+i \infty} s \psi(s) \rho^{-s} d s=-\rho \frac{\partial}{\partial \rho} \int_{\rho}^{1} \frac{g_{1}(t) d t}{\sqrt{\left(t^{2}-\rho^{2}\right)}}
$$

where $g(t)$ is an auxiliary function, as yet unspecified, then it follows from the inversion theorem for Mellin-transform and (1.5) that

$$
s \psi(s)=\frac{s \Gamma\left(\frac{1}{2} s\right) \Gamma\left(\frac{1}{2}\right)}{2 \Gamma\left(\frac{1}{2} s+\frac{1}{2}\right)} \int_{0}^{1} g_{1}(t) t^{s-1} d t, \quad R(s)>-1 .
$$

We rewrite the equation (2.1) as

$$
\frac{1}{2 \pi i} \int_{c-i \infty}^{c+i \infty} \psi(s) \tan \frac{1}{2} \pi s \rho^{-s} d s=F_{1}(\rho), \quad 0<\rho<1,
$$


where

$$
F_{1}(\rho)=f_{1}(\rho)+\frac{1}{2 \pi i} \int_{c-i \infty}^{c+i \infty} \psi(s)\left(\tan \frac{1}{2} \pi s-\tan \alpha s\right) \rho^{-s} d s
$$

Substituting for $\psi(s)$ from (2.3) in the equation (2.4), we obtain, on changing the order of integration, the equation

$$
\int_{0}^{1} \frac{g_{1}(t)}{t} \frac{1}{2 \pi i} \int_{c-i \infty}^{c+i \infty}\left(\frac{t}{\rho}\right)^{s} \frac{\Gamma\left(\frac{1}{2} s\right) \Gamma\left(\frac{1}{2}\right)}{2 \Gamma\left(\frac{1}{2} s+\frac{1}{2}\right)} \tan \frac{1}{2} \pi s d s d t=F_{1}(\rho), \quad 0<\rho<1 .
$$

By considering the integral

$$
\frac{1}{2 \pi i} \int_{C}\left(\frac{t}{\rho}\right)^{s} \frac{\Gamma\left(\frac{1}{2} s\right) \Gamma\left(\frac{1}{2}\right)}{2 \Gamma\left(\frac{1}{2} s+\frac{i}{2}\right)} \tan \frac{1}{2} \pi s d s
$$

where $C$ consists of the sides of the rectangle with vertices $c \pm i T$ and $N \pm i T$, $N$ being an even integer, positive if $t<\rho$ and negative if $t>\rho$, it can be easily shown that

$$
\frac{1}{2 \pi i} \int_{c-i \infty}^{c+i \infty}\left(\frac{t}{\rho}\right)^{s} \frac{\Gamma\left(\frac{1}{2} s\right) \Gamma\left(\frac{1}{2}\right)}{2 \Gamma\left(\frac{1}{2} s+\frac{1}{2}\right)} \tan \frac{1}{2} \pi s d s= \begin{cases}-t\left(\rho^{2}-t^{2}\right)^{-\frac{1}{2}}, & t<\rho \\ 0, & t>\rho .\end{cases}
$$

The equation (2.6) is therefore equivalent to the equation

$$
-\int_{0}^{\rho} \frac{g_{1}(t) d t}{\sqrt{\left(\rho^{2}-t^{2}\right)}}=F_{1}(\rho)
$$

an integral equation of Abel-type whose solution is given by the relation

$$
g_{1}(t)=-\frac{2}{\pi} \frac{d}{d t} \int_{0}^{t} \frac{\rho F_{1}(\rho) d \rho}{\sqrt{\left(t^{2}-\rho^{2}\right)}}
$$

Carrying out the simplification with the help of (1.5), we get the Fredholm equation of the second kind

where

$$
g_{1}(t)=-\frac{2}{\pi} \frac{d}{d t} \int_{0}^{t} \frac{\rho f_{1}(\rho) d \rho}{\sqrt{\left(t^{2}-\rho^{2}\right)}}-\int_{0}^{1} \frac{g(u)}{u} K_{1}(u, t) d u, \quad 0<t<1,
$$

$$
K_{1}(u, t)=\frac{1}{2 \pi i} \int_{c-i \infty}^{c+i \infty}\left(\frac{u}{t}\right)^{s}\left(\tan \frac{1}{2} \pi s-\tan \alpha s\right) \cot \frac{1}{2} \pi s d s,
$$

which may be alternatively written as

$$
K_{1}(u, t)=\frac{1}{2 \pi i} \int_{c-i \infty}^{c+i \infty}\left(\frac{u}{t}\right)^{s} \frac{\sin \left(\frac{1}{2} \pi-\alpha\right) s}{\cos \alpha s \sin \frac{1}{2} \pi s} d s .
$$

In particular if $\alpha=\frac{1}{2} \pi, K_{1}(u, t) \equiv 0$ and the pair of equations (2.1) and (2.2) admits a closed form solution. In every other case, it seems, recourse has to be made to numerical methods for the solution of Fredholm integral equation of the second kind. In fact, for $\alpha=\frac{1}{2} \pi$ all the equations discussed here can be solved analytically and $\psi(s)$ can be determined in a closed form. 
Let us next consider the dual equations

$$
\begin{aligned}
& \frac{1}{2 \pi i} \int_{c-i \infty}^{c+i \infty} \psi(s) \tan \alpha s \rho^{-s} d s=0, \quad 0<\rho<1 \\
& \frac{1}{2 \pi i} \int_{c-i \infty}^{c+i \infty} s \psi(s) \rho^{-s} d s=-f_{2}(\rho), \quad \rho>1 ; 0<c<1 .
\end{aligned}
$$

In this case we begin by assuming that

$$
\frac{1}{2 \pi i} \int_{c-i \infty}^{c+i \infty} \psi(s) \tan \alpha s \rho^{-s} d s=\int_{1}^{\rho} \frac{g_{2}(t) d t}{\sqrt{\left(\rho^{2}-t^{2}\right)}}, \quad \rho>1 .
$$

In view of the inversion theorem for the Mellin-transform and the equation (1.6), this is equivalent to the assumption that

$$
\psi(s) \tan \alpha s=\frac{\Gamma\left(\frac{1}{2}-\frac{1}{2} s\right) \Gamma\left(\frac{1}{2}\right)}{2 \Gamma\left(1-\frac{1}{2} s\right)} \int_{1}^{\infty} g_{2}(t) t^{s-1} d t .
$$

The equation (2.14) can be written in the alternative form

$$
\rho \frac{\partial}{\partial \rho} \frac{1}{2 \pi i} \int_{c-i \infty}^{c+i \infty} \psi(s) \rho^{-s} d s=-f_{2}(\rho), \quad \rho>1,
$$

and if we substitute the value of $\psi(s)$ from the equation (2.16) in (2.17), we get the equation

$\rho \frac{\partial}{\partial \rho}\left[\int_{1}^{\infty} \frac{g_{2}(t)}{t}\left\{\frac{1}{2 \pi i} \int_{c-i \infty}^{c+i \infty}\left(\frac{t}{\rho}\right)^{s} \cot \frac{1}{2} \pi s \frac{\Gamma\left(\frac{1}{2}-\frac{1}{2} s\right) \Gamma\left(\frac{1}{2}\right)}{2 \Gamma\left(1-\frac{1}{2} s\right)} d s\right\} d t\right]=F_{2}(\rho), \quad \rho>1$,

where

$$
F_{2}(\rho)=-f_{2}(\rho)+\int_{1}^{\infty} \frac{g_{2}(t)}{t} \frac{1}{2 \pi i} \int_{c-i \infty}^{c+i \infty} s\left(\cot \frac{1}{2} \pi s-\cot \alpha s\right) \frac{\Gamma\left(\frac{1}{2}-\frac{1}{2} s\right) \Gamma\left(\frac{1}{2}\right)}{2 \Gamma\left(1-\frac{1}{2} s\right)}\left(\frac{t}{\rho}\right)^{s} d s d t
$$

Since

$$
\frac{1}{2 \pi i} \int_{c-i \infty}^{c+i \infty}\left(\frac{t}{\rho}\right)^{s} \cot \frac{1}{2} \pi s \frac{\Gamma\left(\frac{1}{2}-\frac{1}{2} s\right) \Gamma\left(\frac{1}{2}\right)}{2 \Gamma\left(1-\frac{1}{2} s\right)} d s= \begin{cases}t\left(t^{2}-\rho^{2}\right)^{-\frac{1}{2}}, & t>\rho, \\ 0, & t<\rho,\end{cases}
$$

the equation (2.18) is reduced to the equation

$$
\rho \frac{\partial}{\partial \rho} \int_{\rho}^{\infty} \frac{g_{2}(t) d t}{\sqrt{\left(t^{2}-\rho^{2}\right)}}=F_{2}(\rho), \quad \rho>1 .
$$

The solution of the equation (2.21) is

$$
g_{2}(t)=-\frac{2 t}{\pi} \int_{t}^{\infty} \frac{F_{2}(\rho) d \rho}{\sqrt{\left(\rho^{2}-t^{2}\right)}} .
$$

$F_{2}(\rho)$ involves $g_{2}(t)$ but if we carry out the elementary integrations in (2.22) we easily find it to be equivalent to the Fredholm integral equation of the 
second kind

where

$$
g_{2}(t)=\frac{2 t}{\pi} \int_{t}^{\infty} \frac{f_{2}(\rho) d \rho}{\sqrt{\left(\rho^{2}-t^{2}\right)}}-\int_{1}^{\infty} \frac{g_{2}(u)}{u} K_{2}(u, t) d u,
$$

or

$$
K_{2}(u, t)=\frac{1}{2 \pi i} \int_{c-i \infty}^{c+i \infty}\left(\cot \frac{\pi}{2} s-\cot \alpha s\right) \tan \frac{\pi}{2} s\left(\frac{u}{t}\right)^{s} d s
$$

$$
K_{2}(u, t)=\frac{1}{2 \pi i} \int_{c-i \infty}^{c+i \infty}\left(\frac{u}{t}\right)^{s} \frac{\sin \left(\frac{\pi}{2}-\alpha\right) s d s}{\sin \alpha s \cos \frac{\pi}{2} s}
$$

3. Solution of the dual equations

$$
\begin{aligned}
& \frac{1}{2 \pi i} \int_{c-i \infty}^{c+i \infty} s \psi(s) \rho^{-s} d s=-f_{1}(\rho), \quad 0<\rho<1, \\
& \frac{1}{2 \pi i} \int_{c-i \infty}^{c+i \infty} \psi(s) \tan \alpha s \rho^{-s} d s=0, \quad \rho>1 ; \quad-1<c<1,
\end{aligned}
$$

can be accomplished in a similar manner. The starting point in this case is the assumption that

$$
\frac{1}{2 \pi i} \int_{c-i \infty}^{c+i \infty} \psi(s) \tan \alpha s \rho^{-s} d s=\rho \int_{\rho}^{1} \frac{g_{1}(t) d t}{\sqrt{\left(t^{2}-\rho^{2}\right)}}, \quad 0<\rho<1,
$$

which amounts to assuming that

However, since

$$
s \psi(s)=\cot \alpha s \frac{\Gamma\left(\frac{1}{2} s+\frac{1}{2}\right) \Gamma\left(\frac{1}{2}\right)}{\Gamma\left(\frac{1}{2} s\right)} \int_{0}^{1} g_{1}(t) t^{s} d t .
$$

$$
\frac{1}{2 \pi i} \int_{c-i \infty}^{c+i \infty}\left(\frac{t}{\rho}\right)^{s} \frac{\Gamma\left(\frac{1}{2} s+\frac{1}{2}\right)}{\Gamma\left(\frac{1}{2} s\right)} \cot \frac{1}{2} \pi s d s= \begin{cases}-t^{2} \frac{\partial}{\partial \rho}\left(\rho^{2}-t^{2}\right)^{-\frac{1}{2}}, & \rho>t \\ 0, & \rho<t\end{cases}
$$

on substituting the value of $s \psi(s)$ from the equation (3.4) in the equation (3.1) we get the equation

$$
\begin{aligned}
& -\frac{\partial}{\partial \rho} \int_{0}^{\rho} \frac{t^{2} g_{1}(t) d t}{\sqrt{\left(\rho^{2}-t^{2}\right)}} \\
& \quad=-f_{1}(\rho)+\int_{0}^{1} g_{1}(u) \frac{1}{2 \pi i} \int_{c-i \infty}^{c+i \infty}\left(\cot \frac{1}{2} \pi s-\cot \alpha s\right) \frac{\Gamma\left(\frac{1}{2} s+\frac{1}{2}\right) \Gamma\left(\frac{1}{2}\right)}{\Gamma\left(\frac{1}{2} s\right)} \rho^{-s} d s d u .
\end{aligned}
$$

If we regard the right hand side of (3.6) as known for the time being, (3.6) is an integral equation of Abel-type whose solution is given by the relation

$$
\operatorname{tg}_{1}(t)=\frac{2}{\pi} \int_{0}^{t} \frac{f_{1}(\rho) d \rho}{\sqrt{\left(t^{2}-\rho^{2}\right)}}+\int_{0}^{1} g_{1}(u) K_{2}(u, t) d u \text {. }
$$


To solve the equations

$$
\begin{aligned}
& \frac{1}{2 \pi i} \int_{c-i \infty}^{c+i \infty} s \psi(s) \rho^{-s} d s=0, \quad 0<\rho<1, \\
& \frac{1}{2 \pi i} \int_{c-i \infty}^{c+i \infty} \psi(s) \tan \alpha s \rho^{-s} d s=f_{2}(\rho), \quad \rho>1, \quad-1<c<1,
\end{aligned}
$$

we proceed by supposing that

$$
\begin{aligned}
\frac{1}{2 \pi l} \int_{c-i \infty}^{c+i \infty} s \psi(s) \rho^{-s} d s & \equiv-\rho \frac{\partial}{\partial \rho} \frac{1}{2 \pi i} \int_{c-i \infty}^{c+i \infty} \psi(s) \rho^{-s} d s \\
& =-\rho \frac{\partial}{\partial \rho}\left\{\rho \int_{1}^{\rho} \frac{g_{2}(t) d t}{\sqrt{\left(\rho^{2}-t^{2}\right)}}\right\}, \quad \rho>1 .
\end{aligned}
$$

In this case, we find that

$$
\psi(s)=\frac{\Gamma\left(\frac{1}{2}\right) \Gamma\left(-\frac{1}{2} s\right)}{2 \Gamma\left(\frac{1}{2}-\frac{1}{2} s\right)} \int_{1}^{\infty} g_{2}(t) t^{s} d t
$$

Substituting the above value of $\psi(s)$ in the equation (3.9), we are led to the equation

$$
\begin{aligned}
& \int_{1}^{\infty} g_{2}(t) \frac{1}{2 \pi i} \int_{c-i \infty}^{c+i \infty} \frac{\tan \frac{1}{2} \pi s \Gamma\left(\frac{1}{2}\right) \Gamma\left(-\frac{1}{2} s\right)}{2 \Gamma\left(\frac{1}{2}-\frac{1}{2} s\right)}\left(\frac{t}{\rho}\right)^{s} d s d t \\
& \quad=f_{2}(\rho)+\int_{1}^{\infty} g_{2}(u) \frac{1}{2 \pi i} \int_{c-i \infty}^{c+i \infty} \frac{\left(\tan \frac{1}{2} \pi s-\tan \alpha s\right) \Gamma\left(\frac{1}{2}\right) \Gamma\left(-\frac{1}{2} s\right)}{2 \Gamma\left(\frac{1}{2}-\frac{1}{2} s\right)}\left(\frac{u}{\rho}\right)^{s} d s d u .
\end{aligned}
$$

Since

$$
\frac{1}{2 \pi i} \int_{c-i \infty}^{c+i \infty}\left(\frac{t}{\rho}\right)^{s} \frac{\Gamma\left(\frac{1}{2}\right) \Gamma\left(-\frac{1}{2} s\right)}{2 \Gamma\left(\frac{1}{2}-\frac{1}{2} s\right)} \tan \frac{\pi s}{2} d s= \begin{cases}\rho\left(t^{2}-\rho^{2}\right)^{-\frac{1}{2}}, & t>\rho, \\ 0, & t<\rho,\end{cases}
$$

the equation (3.12) is reduced to

$$
\begin{aligned}
& \rho \int_{\rho}^{\infty} \frac{g_{2}(t) d t}{\sqrt{\left(t^{2}-\rho^{2}\right)}} \\
& \quad=f_{2}(\rho)+\int_{1}^{\infty} g_{2}(u) \frac{1}{2 \pi i} \int_{c-i \infty}^{c+i \infty} \frac{\left(\tan \frac{1}{2} \pi s-\tan \alpha s\right) \Gamma\left(-\frac{1}{2} s\right) \Gamma\left(\frac{1}{2}\right)}{2 \Gamma\left(\frac{1}{2}-\frac{1}{2} s\right)}\left(\frac{u}{\rho}\right)^{s} d s d u,
\end{aligned}
$$

which is equivalent to

$$
g_{2}(t)=-\frac{2 t}{\pi} \frac{d}{d t} \int_{t}^{\infty} \frac{f_{2}(\rho) d \rho}{\sqrt{\left(\rho^{2}-t^{2}\right)}}+\int_{1}^{\infty} g_{2}(u) K_{1}(u, t) d u
$$

4. Let us now consider the solution of the equations

$$
\begin{array}{ll}
\frac{1}{2 \pi i} \int_{c-i \infty}^{c+i \infty} \psi(s) \rho^{-s} d s=f_{1}(\rho), & 0<\rho<1, \\
\frac{1}{2 \pi i} \int_{c-i \infty}^{c+i \infty} s \psi(s) \tan \alpha s \rho^{-s} d s=0, & \rho>1 .
\end{array}
$$


Our approach is fruitful in this case only if $-1<c<0$. In terms of the auxiliary function we set, for $0<\rho<1$,

$$
\frac{1}{2 \pi i} \int_{c-i \infty}^{c+i \infty} s \psi(s) \tan \alpha s \rho^{-s} d s=-\rho \frac{\partial}{\partial \rho}\left[\rho \int_{\rho}^{1} \frac{g_{1}(t) d t}{\sqrt{\left(t^{2}-\rho^{2}\right)}}\right] .
$$

This amounts to the assumption that

$$
\psi(s)=\frac{\Gamma\left(\frac{1}{2} s+\frac{1}{2}\right) \Gamma\left(\frac{1}{2}\right)}{2 \Gamma\left(\frac{1}{2} s+1\right)} \cot \alpha s \int_{0}^{1} g_{1}(t) t^{s} d t .
$$

Substituting this value of $\psi(s)$ in the equation (4.1) we get on interchanging the order of integration the equation

$$
\begin{aligned}
& \int_{0}^{1} g(t)\left\{\frac{1}{2 \pi i} \int_{c-i \infty}^{c+i \infty}\left(\frac{t}{\rho}\right)^{s} \cot \frac{1}{2} \pi s \frac{\Gamma\left(\frac{1}{2} s+\frac{1}{2}\right) \Gamma\left(\frac{1}{2}\right)}{2 \Gamma\left(\frac{1}{2} s+1\right)} d s\right\} d t \\
& =f_{1}(\rho)+\int_{0}^{1} g(u)\left\{\frac{1}{2 \pi i} \int_{c-i \infty}^{c+i \infty}\left(\frac{u}{\rho}\right)^{s} \frac{\left(\cot \frac{1}{2} \pi s-\cot \alpha s\right) \Gamma\left(\frac{1}{2} s+\frac{1}{2}\right) \Gamma\left(\frac{1}{2}\right)}{2 \Gamma\left(\frac{1}{2} s+1\right)} d s\right\} d u .
\end{aligned}
$$

However, since

$$
\frac{1}{2 \pi i} \int_{c-i \infty}^{c+i \infty}\left(\frac{t}{\rho}\right)^{s} \cot \frac{1}{2} \pi s \frac{\Gamma\left(\frac{1}{2} s+\frac{1}{2}\right) \Gamma\left(\frac{1}{2}\right)}{2 \Gamma\left(\frac{1}{2} s+1\right)} d s= \begin{cases}\rho\left(\rho^{2}-t^{2}\right)^{-\frac{1}{2}}, & \rho>t, \\ 0, & \rho<t,\end{cases}
$$

the left-hand side of the equation is simplified to

$$
\rho \int_{0}^{\rho} \frac{g(t) d t}{\sqrt{\left(\rho^{2}-t^{2}\right)}}
$$

and therefore the equation (4.5) is reduced to the Fredholm equation of the second kind

$$
g(t)=\frac{2}{\pi} \frac{d}{d t} \int_{0}^{t} \frac{f(\rho) d \rho}{\sqrt{\left(t^{2}-\rho^{2}\right)}}+\frac{1}{t} \int_{0}^{1} g(u) K_{2}(u, t) d u .
$$

To complete the solution of the equations $\left(b_{1}\right)$, we now turn to the dual equations

$$
\begin{aligned}
& \frac{1}{2 \pi i} \int_{c-i \infty}^{c+i \infty} \psi(s) \rho^{-s} d s=0, \quad 0<\rho<1, \\
& \frac{1}{2 \pi i} \int_{c-i \infty}^{c+i \infty} s \psi(s) \tan \alpha s \rho^{-s} d s=f_{2}(\rho), \quad \rho>1 ; \quad-1<c<1 .
\end{aligned}
$$

If we put

$$
\frac{1}{2 \pi i} \int_{c-i \infty}^{c+i \infty} \psi(s) \rho^{-s} d s=\rho \int_{1}^{\rho} \frac{g_{2}(t) d t}{\sqrt{\left(\rho^{2}-t^{2}\right)}}, \quad \rho>1,
$$

we obtain for $\psi(s)$ the representation

$$
\psi(s)=\frac{\Gamma\left(-\frac{1}{2} s\right) \Gamma\left(\frac{1}{2}\right)}{2 \Gamma\left(\frac{1}{2}-\frac{1}{2} s\right)} \int_{1}^{\infty} g_{2}(t) t^{s} d t
$$


which has the property that the equation (4.9) is identically satisfied. Replacing: $\psi(s)$ in the equation (4.10) by its value given by the relation (4.12), we find that.

$$
\begin{aligned}
& -\rho \frac{\partial}{\partial \rho}\left[\int_{1}^{\infty} g_{2}(t) \frac{1}{2 \pi i} \int_{c-i \infty}^{c+i \infty}\left(\frac{\rho}{t}\right)^{-s} \frac{\Gamma\left(-\frac{1}{2} s\right) \Gamma\left(\frac{1}{2}\right)}{2 \Gamma\left(\frac{1}{2}-\frac{1}{2} s\right)} \tan \frac{\pi}{2} s d s d t\right] \\
& =f_{2}(\rho)+\int_{1}^{\infty} g_{2}(u) \frac{1}{2 \pi i} \int_{c-i \infty}^{c+i \infty}\left(\frac{\rho}{u}\right)^{-s} \frac{\Gamma\left(-\frac{1}{2} s\right) \Gamma\left(\frac{1}{2}\right)}{2 \Gamma\left(\frac{1}{2}-\frac{1}{2} s\right)}\left(\tan \frac{\pi}{2} s-\tan \alpha s\right) d s d u .
\end{aligned}
$$

In view of the relation (3.13), the above equation is reduced to

$$
\begin{aligned}
& -\rho \frac{\partial}{\partial \rho} \rho \int_{\rho}^{\infty} \frac{g_{2}(t) d t}{\sqrt{\left(t^{2}-\rho^{2}\right)}} \\
& \quad=f_{2}(\rho)+\int_{1}^{\infty} g_{2}(u) \frac{1}{2 \pi i} \int_{c-i \infty}^{c+i \infty} s\left(\frac{\rho}{u}\right)^{-s} \frac{\Gamma\left(\frac{1}{2}\right) \Gamma\left(-\frac{1}{2} s\right)}{2 \Gamma\left(\frac{1}{2}-\frac{1}{2} s\right)}\left(\tan \frac{\pi}{2} s-\tan \alpha s\right) d s d u .
\end{aligned}
$$

If we treat the right-hand side as known, then the equation (4.14) is an Abeltype of integral equation, whose solution is found to be

$$
g_{2}(t)=-\frac{2}{\pi t} \int_{t}^{\infty} \frac{f_{2}(u) d u}{\sqrt{\left(u^{2}-t^{2}\right)}}+\frac{1}{t} \int_{1}^{\infty} g_{2}(u) K_{1}(u, t) d u .
$$

5. Finally, let us consider the equations $\left(b_{2}\right)$. For the equations

$$
\begin{aligned}
& \frac{1}{2 \pi i} \int_{c-i \infty}^{c+i \infty} s \psi(s) \tan \alpha s \rho^{-s} d s=f_{1}(\rho), \quad 0<\rho<1, \\
& \frac{1}{2 \pi i} \int_{c-i \infty}^{c+i \infty} \psi(s) \rho^{-s} d s=0, \quad \rho>1 ; \quad-1<c<1,
\end{aligned}
$$

the relevant assumption is that for $0<\rho<1$

$$
\frac{1}{2 \pi i} \int_{c-i \infty}^{c+i \infty} \psi(s) \rho^{-s} d s=\int_{\rho}^{1} \frac{g_{1}(t) d t}{\sqrt{\left(t^{2}-\rho^{2}\right)}}
$$

This corresponds to the assumption that

$$
\psi(s)=\frac{\Gamma\left(\frac{1}{2} s\right) \Gamma\left(\frac{1}{2}\right)}{2 \Gamma\left(\frac{1}{2} s+\frac{1}{2}\right)} \int_{0}^{1} g_{1}(t) t^{s-1} d t .
$$

Since the first equation is equivalent to

$$
-\rho \frac{\partial}{\partial \rho} \frac{1}{2 \pi i} \int_{c-i \infty}^{c+i \infty} \psi(s) \tan \alpha s \rho^{-s} d s=f_{1}(\rho), \quad 0<\rho<1,
$$

on substituting for $\psi(s)$ and interchanging the order of integration we obtain. 
the equation

$$
\begin{aligned}
& -\rho \frac{\partial}{\partial \rho} \int_{0}^{1} \frac{g_{1}(t)}{t}\left\{\frac{1}{2 \pi i} \int_{c-i \infty}^{c+i \infty} \frac{\Gamma\left(\frac{1}{2} s\right) \Gamma\left(\frac{1}{2}\right)}{2 \Gamma\left(\frac{1}{2} s+\frac{1}{2}\right)}\left(\frac{t}{\rho}\right)^{s} \tan \frac{1}{2} \pi s d s\right\} d t \\
& =f_{1}(\rho)+\int_{0}^{1} \frac{g_{1}(u)}{u} \cdot \frac{1}{2 \pi i} \int_{c-i \infty}^{c+i \infty} s \psi(s) \frac{\Gamma\left(\frac{1}{2} s\right) \Gamma\left(\frac{1}{2}\right)}{2 \Gamma\left(\frac{1}{2} s+\frac{1}{2}\right)}\left(\frac{u}{\rho}\right)^{s}\left(\tan \frac{1}{2} \pi s-\tan \alpha s\right) d s d u .
\end{aligned}
$$

From the relation (2.7) it therefore follows that

$$
\begin{aligned}
& \rho \frac{\partial}{\partial \rho} \int_{0}^{\rho} \frac{t g_{1}(t) d t}{\sqrt{\left(\rho^{2}-t^{2}\right)}} \\
& \quad=f_{1}(\rho)+\int_{0}^{1} \frac{g_{1}(u)}{u} \frac{1}{2 \pi i} \int_{c-i \infty}^{c+i \infty} s \psi(s) \frac{\Gamma\left(\frac{1}{2} s\right) \Gamma\left(\frac{1}{2}\right)}{2 \Gamma\left(\frac{1}{2} s+\frac{1}{2}\right)}\left(\frac{u}{\rho}\right)^{s}\left(\tan \frac{\pi}{2} s-\tan \alpha s\right) d s d u,
\end{aligned}
$$

an equation from which it is easily deduced that $g_{1}(t)$ satisfies the integral equation

$$
g_{1}(t)=\frac{2}{\pi} \int_{0}^{t} \frac{f_{1}(\rho) d \rho}{\rho \sqrt{\left(t^{2}-\rho^{2}\right)}}+\frac{1}{t} \int_{0}^{1} \frac{g_{1}(u)}{u} K_{1}(u, t) d u .
$$

We conclude our discussion of dual integral equations by describing the solution of the equations

$$
\begin{aligned}
& \frac{1}{2 \pi i} \int_{c-i \infty}^{c+l \infty} s \psi(s) \tan \alpha s \rho^{-s} d s=0, \quad 0<\rho<1, \\
& \frac{1}{2 \pi i} \int_{c-i \infty}^{c+i \infty} \psi(s) \rho^{-s} d s=f_{2}(\rho), \quad \rho>1,
\end{aligned}
$$

for $0<c<1$. The equation (5.9) is in fact equivalent to the equation

$$
\rho \frac{\hat{o}}{\partial \rho} \frac{1}{2 \pi i} \int_{c-i \infty}^{c+i \infty} \psi(s) \tan \alpha s \rho^{-s} d s=0, \quad 0<\rho<1 .
$$

If we assume that

$$
\frac{1}{2 \pi i} \int_{c-i \infty}^{c+i \infty} \psi(s) \tan \alpha s \rho^{-s} d s= \begin{cases}0, & 0<\rho<1, \\ \int_{1}^{\rho} \frac{g(t) d t}{\sqrt{\left(\rho^{2}-t^{2}\right)}}, & \rho>1,\end{cases}
$$

it is easily shown that

$$
\psi(s)=\frac{\Gamma\left(\frac{1}{2}-\frac{1}{2} s\right) \Gamma\left(\frac{1}{2}\right)}{2 \Gamma\left(1-\frac{1}{2} s\right)} \cot \alpha s \int_{1}^{\infty} g(t) t^{s-1} d t
$$


Substituting the above value of $\psi(s)$ in the equation (5.10) and interchanging the order of integration, we get the relation

$$
\begin{aligned}
& \int_{1}^{\infty} \frac{g(t)}{t} \frac{1}{2 \pi i} \int_{c-i \infty}^{c+i \infty} \cot \frac{1}{2} \pi s \frac{\Gamma\left(\frac{1}{2}-\frac{1}{2} s\right) \Gamma\left(\frac{1}{2}\right)}{2 \Gamma\left(1-\frac{1}{2} s\right)}\left(\frac{t}{\rho}\right)^{s} d s d t \\
& \quad=f_{2}(\rho)+\int_{1}^{\infty} \frac{g(u)}{u} \cdot \frac{1}{2 \pi i} \int_{c-i \infty}^{c+i \infty} \frac{\left(\cot \frac{1}{2} \pi s-\cot \alpha s\right) \Gamma\left(\frac{1}{2}-\frac{1}{2} s\right) \Gamma\left(\frac{1}{2}\right)(u / \rho)^{s}}{2 \Gamma\left(1-\frac{1}{2} s\right)} d s d u .
\end{aligned}
$$

From the relation (2.20), it follows that

$$
\begin{aligned}
& \int_{\rho}^{\infty} \frac{g(t) d t}{\sqrt{\left(t^{2}-\rho^{2}\right)}} \\
& \quad=f_{2}(\rho)+\int_{1}^{\infty} \frac{g(u)}{u} \cdot \frac{1}{2 \pi i} \int_{c-i \infty}^{c+i \infty} \frac{\left(\cot \frac{1}{2} \pi s-\cot \alpha s\right) \Gamma\left(\frac{1}{2}-\frac{1}{2} s\right) \Gamma\left(\frac{1}{2}\right)(u / \rho)^{s}}{2 \Gamma\left(1-\frac{1}{2} s\right)} d s d u
\end{aligned}
$$

which implies that

$$
g(t)=-\frac{2}{\pi} \frac{d}{d t} \int_{t}^{\infty} \frac{\rho f_{2}(\rho) d \rho}{\sqrt{\left(\rho^{2}-t^{2}\right)}}+\int_{1}^{\infty} \frac{g(u)}{u} K_{2}(u, t) d u .
$$

\section{Kernels of the integral equations}

It is possible to write $K_{1}(u, t)$ and $K_{2}(u, t)$ in terms of the infinite series. For example, for $u<t$ we obtain by moving the line of integration to $R(s)=\infty$

$$
K_{1}(u, t)=\frac{2}{\pi} \sum_{n=1}^{\infty}\left(\frac{u}{t}\right)^{2 n} \tan 2 n \alpha-\frac{1}{\alpha} \sum_{n=1}^{\infty}\left(\frac{u}{t}\right)^{2 n} \cot \frac{(2 n+1) \pi^{2}}{4 \alpha}
$$

and if $u>t$, then shifting the line of integration to $R(s)=-\infty$, we find that

$$
K_{1}(u, t)=\frac{2}{\pi} \sum_{n=1}^{\infty}\left(\frac{t}{u}\right)^{2 n} \tan 2 n \alpha-\frac{1}{\alpha} \sum_{n=1}^{\infty}\left(\frac{t}{u}\right)^{2 n} \cot \frac{(2 n+1) \pi^{2}}{4 \alpha} .
$$

The calculations leading to the above results are based on the assumption that for $n o$ integral values of $m$ and $n$ is $\alpha=(2 m+1) \pi / 4 n$. This ensures that all the poles are simple poles; otherwise account has to be taken of multiple poles.

Similarly for $u<t$,

$$
K_{2}(u, t)=\frac{2}{\pi} \sum_{n=0}^{\infty}\left(\frac{u}{t}\right)^{2 n+1} \cot (2 n+1) \alpha-\frac{1}{\alpha} \sum_{n=1}^{\infty}\left(\frac{u}{t}\right)^{n} \tan \frac{n \pi^{2}}{2 \alpha}
$$

and for $u>t$,

$$
K_{2}(u, t)=\frac{2}{\pi} \sum_{n=0}^{\infty}\left(\frac{u}{t}\right)^{-2 n-1} \cot (2 n+1) \alpha-\frac{1}{\alpha} \sum_{n=1}^{\infty}\left(\frac{t}{u}\right)^{n} \tan \frac{n \pi^{2}}{2 \alpha} .
$$

There is an apparent singularity at $t=u$ in both the kernels because the power series representations seem to diverge but as is obvious from the integral, $K_{1}$ and $K_{2}$ are bounded for all positive real values of $u$ and $t$. For computational 
purposes, however, it seems to be best to replace the complex integral by a real line integral and since the integrand involves only elementary functions, the value of the integral can be easily obtained by numerical integration.

Shifting the line of integration to the imaginary axis, it is readily shown that

and that

$$
K_{\mathrm{t}}(u, t)=\frac{1}{\pi} \int_{0}^{\infty} \frac{\sinh \left(\frac{1}{2} \pi-\alpha\right) y \cos (y \log u / t) d y}{\cosh \alpha y \sinh \frac{1}{2} \pi y}
$$

$$
K_{2}(u, t)=\frac{1}{\pi} \int_{0}^{\infty} \frac{\sinh \left(\frac{1}{2} \pi-\alpha\right) y \cos (y \log u / t)}{\sinh \alpha y \cosh \frac{1}{2} \pi y} d y
$$

\section{REFERENCES}

(1) I. N. SNEDdon and R. P. SRIVASTAv, Dual series relations-I, Dual relations involving Fourier Bessel series, Proc. Roy. Soc. Edin. A66 (1964), 150-160.

(2) E. C. Titchmarsh, Introduction to the Theory of Fourier Integrals (Oxford, 1948), p. 46.

Department of Mathematics

INDIAN INSTITUTE OF TECHNOLOGY

KANPUR, U.P., INDIA 